ISSN 1112-9867

http://www.jfas.info

\title{
PERFORMANCE SIMULATION OF PARABOLIC TROUGH SOLAR COLLECTOR USING TWO FLUIDS (THERMIC OIL AND MOLTEN SALT)
}

\author{
T.E. Boukelia ${ }^{1, *}$, M.S. Mecibah ${ }^{1}$ and A. Laouafi ${ }^{2}$ \\ ${ }^{1}$ Laboratory of Mechanics, Mechanical Engineering Department, Faculty of Technology \\ Sciences, University of Brothers Mentouri, Constantine 25000, Algeria \\ ${ }^{2}$ Department of Electrical Engineering, University of 20 August 1955, Skikda, Algeria
}

Received: 22 Jaunary 2016 / Accepted: 27 April 2016 / Published online: 01 May 2016

\begin{abstract}
The Parabolic trough solar collector is considered as one of the most proven, mature and commercial concentrating solar systems implemented in arid and semi-arid regions for energy production. It focuses sunlight onto a solar receiver by using mirrors and is finally converted to a useful thermal energy by means of a heat transfer fluid. The aims of this study are (i) to develop a new methodology for simulation and performance evaluation of parabolic trough solar collector, in addition (ii) to compare the efficiencies of this system using two different fluids; thermic oil and molten solar salt. The validation of obtained results using this methodology shows a good agreement with those obtained by the experimental tests. Furthermore, this study favors the using of thermic oil as heat transfer fluid in the receiver instead of molten salt due to the high efficiency of the concentrator based on the first fluid in comparison to the second one.
\end{abstract}

Keywords: parabolic trough collector; thermic oil; molten salt; performance.

Author Correspondence, e-mail: taqy25000@ hotmail.com doi: http://dx.doi.org/10.4314/jfas.v8i2.28 


\section{INTRODUCTION}

Un concentrateur solaire cylindro-parabolique $(\mathrm{CCP})$ prend l'énergie rayonnante du soleil et la convertit en énergie thermique utile par l'intermédiaire du fluide de transfert de chaleur (FTC) qui circule dans le champ solaire. Une fois que la géométrie et les propriétés optiques et thermiques sont définies, les performances du CCP peuvent être calculées sous des différentes configurations et conditions météorologiques. Les CCP sont généralement exploités jusqu'à des températures allant à $400{ }^{\circ} \mathrm{C}$ avec l'huile synthétique comme FTC, alors qu'ils peuvent atteindre $550{ }^{\circ} \mathrm{C}$ en utilisant le sel fondu. L'analyse optique et thermique de ces collecteurs est très importante pour le calcul des pertes optiques et thermiques et permet aussi d'évaluer les effets de la dégradation du collecteur [1].

La prédiction des performances optique et thermique des concentrateurs est essentielle pour la conception et l'évaluation de la performance des centrales solaires thermiques à concentration. Dans les dernières années, des études ont été présentées pour évaluer la performance du concentrateur solaire cylindro-parabolique, en supposant que la distribution de la température est uniforme autour de la circonférence du tube récepteur, et en négligeant les influences de la distribution non uniforme du flux d'énergie solaire sur le tube [2-5]. Récemment, les auteurs de la Réf. [6] ont rapporté les résultats de la simulation numérique en 3-dimensions du récepteur lorsque les distributions non-uniformes du flux d'énergie solaire sont considérées. Cheng et al. [7] ont présenté une simulation numérique d'un concentrateur solaire cylindro-parabolique avec la condition du flux solaire non uniforme, par le couplage de la méthode des volumes finis et la méthode de Monte Carlo (Ray-Trace), dans laquelle les effets des propriétés des différents FTC sur les distributions de température dans le récepteur, les pertes thermiques et le rendement du concentrateur ont été étudiés. He et al. [8] ont utilisé la méthode de Monte Carlo Ray-Trace (MCRT) pour évaluer la distribution du flux solaire incident sur le tube récepteur d'un concentrateur cylindro-parabolique de type LS-2. Cette méthode emploie des mouvements aléatoires de photons, et à chaque réflexion ou transmission, elle juge si le photon frappe le tube récepteur. Tandis que Xiao et al. [9] ont simulé la distribution du flux solaire sur la surface extérieure du tube absorbeur d'un récepteur du concentrateur solaire cylindro-parabolique sur la base de la méthode de Monte Carlo 
Ray-Trace. La distribution du flux non linéaire a été considérée comme une condition aux limites complexe utilisée pour simuler le problème de transfert de chaleur dans ce système.

Néanmoins, aucune étude comparative n'a été trouvée dans la littérature basée sur l'analyse du concentrateur solaire cylindro-parabolique avec deux fluides différents (huile thermique et sel fondu). Par suite, et dans ce travail, nous utilisons une méthodologie pour évaluer les performances optiques et thermiques d'un concentrateur solaire cylindro-parabolique avec deux fluides caloporteurs différents; le premier est l'huile thermique de type Therminol VP1, tandis que le deuxième est le sel fondu $\left(60 \% \mathrm{NaNO}_{3}+40 \% \mathrm{KNO}_{3}\right)$. La deuxième nouveauté de cette étude est de développer un nouveau modèle mathématique pour simuler la performance thermique du tube récepteur d'un concentrateur de même type.

\section{MODELISATION DU CONCENTRATEUR}

\subsection{Description du système}

Dans cette partie de notre étude, la quantité d'énergie captée, et l'analyse des performances optiques et thermiques du concentrateur cylindro-parabolique avec deux fluides caloporteurs (huile thermique et sel fondu) seront investiguées. Le modificateur de l'angle d'incidence, le facteur d'ombrage et la perte d'extrémité, en plus de la perte thermique et l'efficacité de la conversion de l'énergie solaire en énergie thermique à tout moment peuvent être calculés avec le modèle développé dans cette étude pour un concentrateur solaire avec un collecteur Solargenix LS-2 et un récepteur Schott PTR 2008 (Tableau 1), le concentrateur est installé sur un système de poursuite solaire avec un axe horizontal nord-sud et peut suivre le soleil de l'est à l'ouest (Fig 1). Le choix d'un tel concentrateur est motivé par la forte résistance à des gammes de températures élevées et des conditions climatiques difficiles. La simulation de ces paramètres a été effectuée à Béchar (latitude $31,38^{\circ} \mathrm{N}$, longitude $2,15^{\circ} \mathrm{W}$, altitude $806 \mathrm{~m}$ ). 


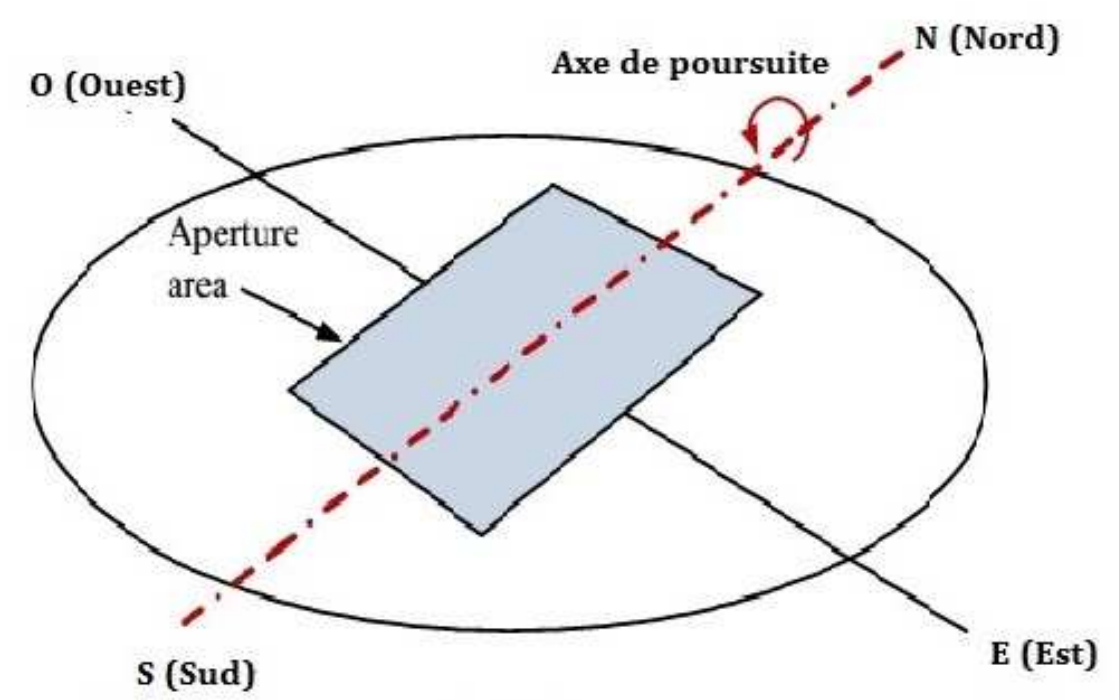

Fig.1. Système de poursuite considéré dans l'étude

Tableau 1. Détails techniques du concentrateur considéré dans l'étude [10-11]

\begin{tabular}{lcc}
\hline & Tube récepteur & Collecteur \\
\hline & Schott PTR 2008 & Solargenix LS-2 \\
\hline Largeur d'ouverture $(m)$ & -- & 5 \\
\hline Longueur d'assemblage du collecteur $(m)$ & -- & 100 \\
\hline Distance focale $(m)$ & -- & 1,8 \\
\hline Diamètre intérieur du tube $(m)$ & 0,066 & -- \\
\hline Diamètre extérieur du tube $(m)$ & 0,070 & -- \\
\hline Diamètre intérieur de l'enveloppe de verre $(m)$ & 0,115 & -- \\
\hline Diamètre extérieur de l'enveloppe de verre $(m)$ & 0,120 & -- \\
\hline
\end{tabular}

\subsubsection{Irradiation interceptée par le concentrateur}

Pour la conception du capteur solaire à concentration, l'estimation du rayonnement solaire direct horaire reçu est essentielle pour le calcul de la performance de ce système tout le long de l'année. Les modèles à long terme prévoient la répartition horaire moyenne du rayonnement solaire global et diffus au cours de la journée moyenne de chaque mois. Compte tenu de l'irradiation globale et diffuse quotidienne moyenne sur une surface horizontale à long terme, il est possible de trouver le rayonnement solaire direct horaire [12]. La procédure qui calcule l'irradiation solaire interceptée par l'ouverture du concentrateur solaire avec les quatre différents modes de poursuite solaire suit ces étapes:

I. Estimation de la déclinaison solaire $\delta$, et de l'angle horaire du coucher du soleil $\omega_{s}[13]$ : 


$$
\begin{aligned}
& \delta=23,45 \sin \left[\frac{(J+284) 360}{365}\right] \\
& \omega_{s}=\arccos [-\tan (\varphi) \tan (\delta)]
\end{aligned}
$$

Ou: $\varphi$ est la Latitude, et $J$ est le numéro du jour de l'année.

II. Estimation de la moyenne mensuelle du rayonnement extraterrestre quotidien $H_{0}$, et la durée d'ensoleillement maximale $S_{0}[13]$ :

$$
\begin{aligned}
& H_{0}=\left(\frac{24 \times 3600 \times I_{s \sigma}}{\pi}\right) d_{r}\left[\cos (\varphi) \cos (\delta) \sin \left(\omega_{s}\right)+\frac{\pi \omega_{s}}{180} \sin (\varphi) \sin (\delta)\right] \\
& S_{0}=\frac{12 \omega_{s}}{15}
\end{aligned}
$$

Avec $\mathrm{I}_{\mathrm{sc}}=0,082 \mathrm{MJ} / \mathrm{m}^{2} / \mathrm{min}\left(1367 \mathrm{~W} / \mathrm{m}^{2}\right)$ est la constante solaire.

III. Estimation de la moyenne mensuelle du rayonnement global quotidien sur un plan horizontal $H_{G}[14]$ :

$$
H_{G}=\left(0,57089+0,01028\left(\frac{S}{S_{0}}\right)-0,00005\left(\frac{S}{S_{0}}\right)^{2}\right) \times H_{0}
$$

IV. Estimation de la moyenne mensuelle du rayonnement diffus quotidien sur un plan horizontal $H_{D}[15]$ :

$$
H_{D}=\left(0,337-0,068\left(\frac{H_{G}}{H_{0}}\right)+0,025\left(\frac{H_{G}}{H_{0}}\right)^{2}-0,002\left(\frac{H_{G}}{H_{0}}\right)^{3}\right) \times H_{G}
$$

V. Estimation de la moyenne mensuelle du rayonnement global horaire sur un plan horizontal [16]:

$$
\begin{aligned}
& l_{G}=r_{t} \times H_{G} \\
& r_{t}=\frac{\pi}{24}(a+b \times \cos \omega)\left(\frac{\cos \omega-\cos \omega_{g}}{\sin \omega_{s}-\left(\pi \omega_{s} / 180\right) \cos \omega_{s}}\right)
\end{aligned}
$$

Avec: $\quad a=0,4090-0,5016 \sin \left(\omega_{s}+1,047\right)$

$$
b=0,6609+0,4767 \sin \left(\omega_{s}+1,047\right)
$$

VI. Estimation de la moyenne mensuelle du rayonnement diffus horaire sur un plan horizontal [17]: 


$$
\begin{aligned}
& I_{D}=r_{d} \times H_{D} \\
& r_{d}=\frac{\pi}{24}\left(\frac{\cos \omega-\cos \omega_{s}}{\sin \omega_{s}-\left(\pi \omega_{s} / 180\right) \cos \omega_{s}}\right)
\end{aligned}
$$

VII. Estimation de la moyenne mensuelle du rayonnement direct horaire sur un plan horizontal:

$$
I_{B H}=I_{G}-I_{D}
$$

I. Estimation de la quantité d'irradiation solaire directe horaire interceptée par l'ouverture du concentrateur [12]:

$$
I_{E}=I_{E H} \frac{\cos \theta_{i}}{\sin \alpha}
$$

Avec $\theta_{i}$ - l'angle d'incidence, qui dépend du mode de la poursuite et de la position du soleil, il est calculé comme suit [18]:

$$
\cos \theta_{i}=\sqrt{\sin ^{2} \alpha+\cos ^{2} \delta \sin ^{2} h}
$$

où: $h$ est l'angle horaire du soleil, Il est calculé comme suite [13.]:

$$
h=15^{\circ}\left(t_{s}-12\right)
$$

Où : $t_{\mathrm{s}}$ est le temps solaire vrai en heures, il est donné par :

$$
t_{s}=t+E O T+\left(l_{s t}-l_{\text {tocat }}\right) \frac{4 m i n}{\text { degree }}
$$

Où $l_{\text {local }}$ est le temps légal ou temps local standard, donné par une montre; $l_{s t}$ est le décalage horaire par rapport au méridien de Greenwich (égal 1 pour l'Algérie); EOT (min) est l'équation du temps, elle est donnée par Woolf [19]:

$$
\begin{aligned}
& E O T=0,258 \cos (x)-7,416 \sin (x)-3,648 \cos (2 x)-9,228 \sin (2 x) \\
& x=\frac{360(J-1)}{365,242}
\end{aligned}
$$

où $\alpha$ est la hauteur angulaire du soleil, elle est donné par l'équation suivante [19]:

$$
\sin \alpha=\sin \delta \times \sin \varphi+\cos \delta \times \cos \varphi \times \cos h
$$




\subsubsection{Modificateur de l'angle d'incidence}

En plus des pertes dues à l'angle d'incidence, Il existe d'autres pertes sur des collecteurs qui peuvent être corrélées à l'angle d'incidence. Ces pertes sont dues à la réflexion et l'absorption additionnelles de l'enveloppe de verre lorsque l'angle d'incidence augmente [18]. Le modificateur de l'angle d'incidence (IAM) corrige ces pertes additionnelles de réflexion et d'absorption, il est donné comme suit [10]:

$$
I A M=1+0,000884 \cdot \frac{\theta_{i}}{\cos \theta_{i}}-0,00005369 \cdot \frac{\theta_{i}^{2}}{\cos \theta_{i}}
$$

\subsubsection{Perte d'extrémité}

Les pertes d'extrémité se produisent au niveau des extrémités du récepteur (Fig 2), où, pour un angle d'incidence non nul, certaine longueur du tube absorbeur n'est pas illuminée par le rayonnement solaire réfléchi par le miroir. La perte d'extrémité est fonction de la distance focale du collecteur, de la longueur du collecteur et de l'angle d'incidence, et est donnée par [20]:

$$
P_{\text {Extrémité }}=1-f \frac{\tan \theta_{i}}{L_{S C A}}
$$

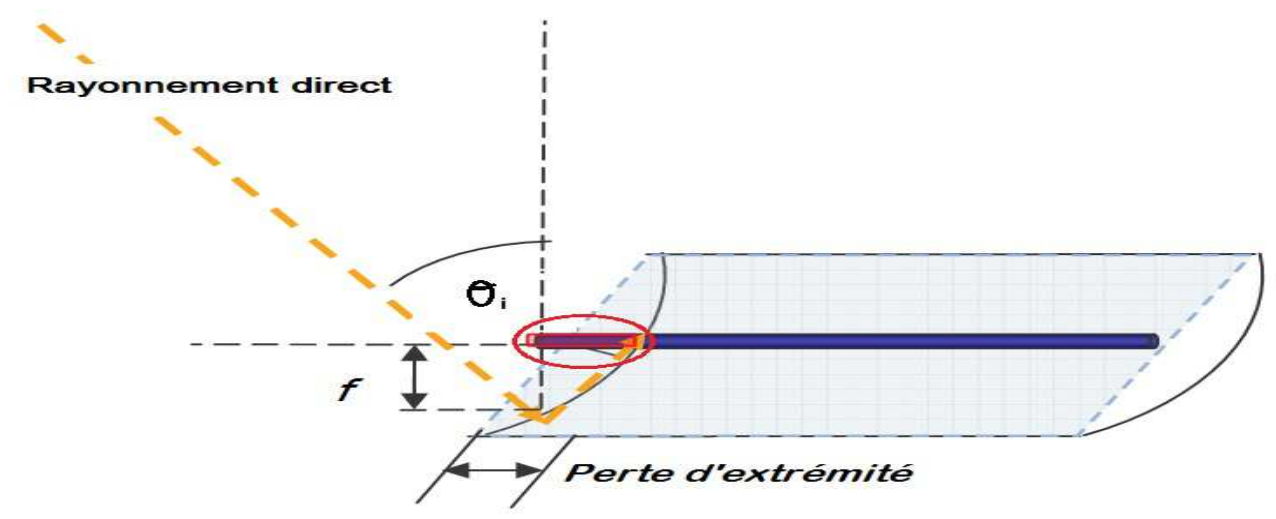

Fig.2. Perte d'extrémité par le tube récepteur [21]

\subsubsection{Distribution non-uniforme du flux solaire sur le récepteur}

La simulation de la performance optique et de la distribution du rayonnement solaire autour du tube récepteur du concentrateur, dans notre travail, a été réalisée à l'aide du logiciel SolTrace, développé au sein de "National Renewable Energy Laboratory (NREL)" pour analyser et simuler les performances optiques et géométriques des systèmes solaires [22]. Le 
code utilise l'approche dite «Lancer des rayons » basée sur la méthode de Monte Carlo.

Lorsque l'utilisateur indique un certain nombre de rayons qui doivent être tracés, afin de mener les calculs optiques, de différentes simulations ont été réalisées en faisant varier ce paramètre. Pour cette étude, les simulations ont été réalisées avec 2.500 .000 rayons issus du disque solaire lancés en direction du concentrateur avec un flux solaire direct normal égal à $1000 \mathrm{~W} / \mathrm{m}^{2}$. La cartographie des points d'impact des rayons solaires sur les différents éléments du système optique a été obtenue, à partir de cette dernière, on détermine le flux solaire collecté par les deux différents composants; réflecteur et récepteur (Fig 3).
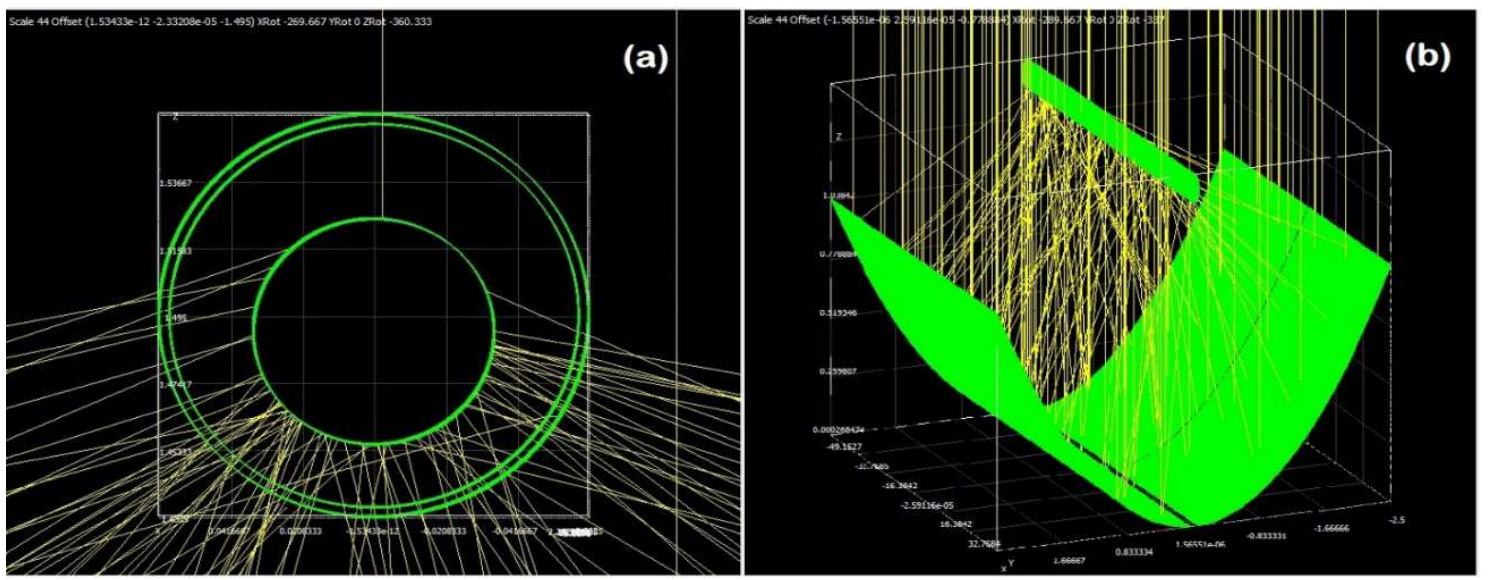

Fig.3. Intersection des rayons avec (a) le récepteur, (b) le réflecteur

Pour un PC qui a les propriétés suivantes :

- Processeur: Intel (R) Core (TM) i5-5200 U CPU @2,20 GHz

- Mémoire installée (RAM) 6,00.

- Carte graphique: Intel (R) HD Graphics 5500.

La simulation de la distribution du flux solaire au niveau de l'absorbeur prend 356,8 secondes pour 2.500.000 itérations.

La figure 4 montre la distribution du flux solaire le long de la paroi extérieure du tube absorbeur en tenant compte de la géométrie cylindro-parabolique de notre concentrateur de type LS-2. Comme représentée sur la figure 3, en plus de la figure 4, la courbe peut être divisée en quatre parties détaillées comme suit: Dans la première partie, le flux de chaleur solaire est très faible, puisque le tube absorbeur ne reçoit que le rayonnement direct. Dans la deuxième partie, le flux solaire augmente rapidement et atteint un pic d'environ $48.765 \mathrm{~W} / \mathrm{m}^{2}$. 
Il est montré que le flux de chaleur du rayonnement solaire concentré atteint la périphérie inférieure du tube absorbeur rapidement. La distribution non uniforme du flux d'énergie solaire conduit à une distribution non uniforme de la température, ce qui amène des contraintes mécaniques dans le récepteur. Ensuite, le résultat (la distribution du flux d'énergie solaire) est considéré comme une des conditions aux limites du flux de chaleur dans le modèle de simulation de la performance thermique.

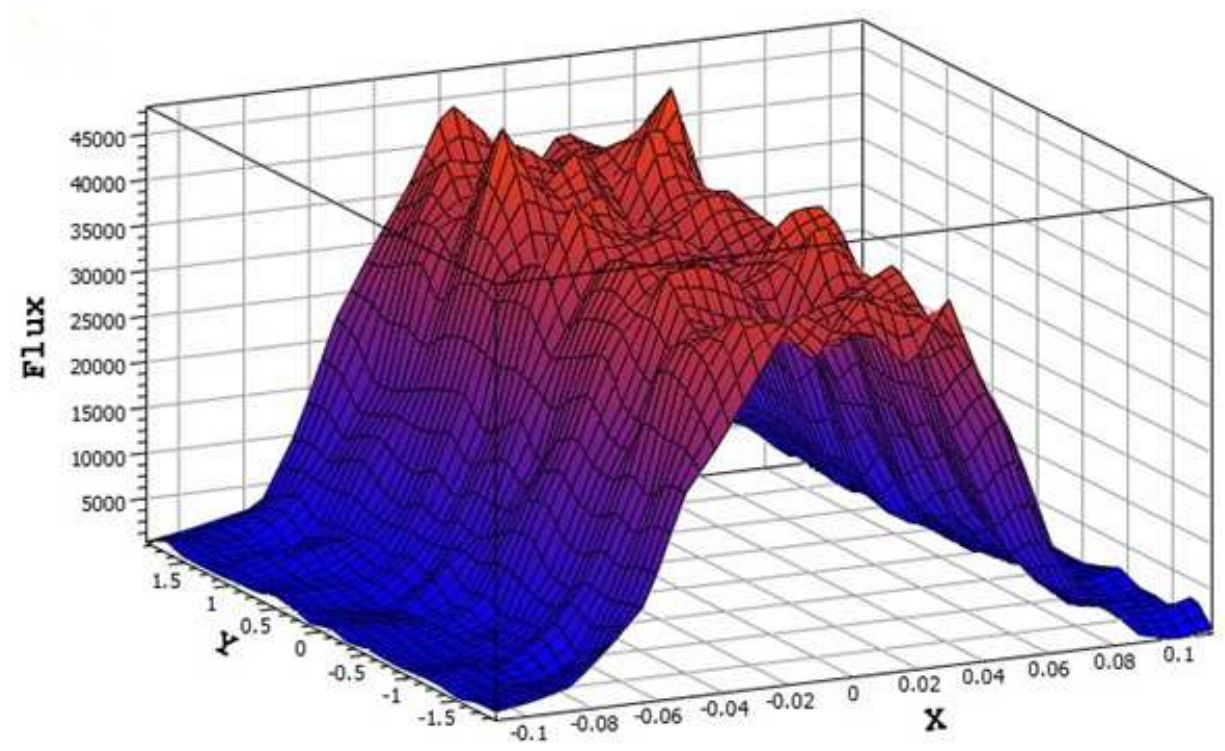

Fig.4. Distribution moyenne du flux thermique au niveau de l'absorbeur avec IND =1000 $\left[\mathrm{W} / \mathrm{m}^{2}\right]$ en $3 \mathrm{D}$

\subsubsection{Performance thermique du récepteur}

Une modélisation mathématique du transfert de chaleur a été construite pour simuler tous les flux de chaleurs et tous les bilans énergétiques pour chacune des surfaces du récepteur solaire de type Schott 2008 PTR. Ce modèle est basé sur une étude précédente présentée par J.L Moreno [23], et a été modifié selon notre problème pour évaluer les performances thermiques de notre système en utilisant deux fluides caloporteurs différents: l'huile thermique (Therminol VP-1) et le sel fondu solaire. Les fluides de transfert de chaleur travaillent avec deux intervalles de température; entre 0 à $400{ }^{\circ} \mathrm{C}$ et de 220 à $550{ }^{\circ} \mathrm{C}$ pour l'huile et le sel, respectivement. Ces dernières températures étant limitées par les propriétés des FTCs, ces propriétés ont également été examinées à l'annexe II. Puisque toutes les propriétés physiques de chacun des éléments du concentrateur solaire sont connues, ainsi que les propriétés optiques, 
en plus de la distribution du flux sur le récepteur étaient déjà simulées, le bilan énergétique peut être écrit pour chaque surface. Le schéma de transfert de chaleur de notre concentrateur solaire cylindro-parabolique est présenté dans les figures 5 et 6 .

En tenant compte de tous les processus de transfert de chaleur dans notre modèle unidimensionnel (1-D) comme illustré dans la figure suivante 6 , quatre équations venant du bilan énergétique à chacune des surfaces peuvent être écrites comme suit:

$$
\begin{aligned}
& \dot{q}_{1-\text { convection }}=\dot{q}_{1-\text { conduction }} \\
& \dot{q}_{1 \text {-absorption solaire }}=\dot{q}_{2 \text {-convection }}+\dot{q}_{1 \text {-rayonnement }}+\dot{q}_{1-c o n d u c t i o n} \\
& \dot{q}_{2 \text {-convection }}+\dot{q}_{1 \text {-rayonnement }}=\dot{q}_{2 \text {-conduction }} \\
& \dot{q}_{2 \text {-conduction }}+\dot{q}_{2 \text {-absorption sola ire }}=\dot{q}_{3 \text {-convection }}+\dot{q}_{2 \text {-rayonnement }}
\end{aligned}
$$

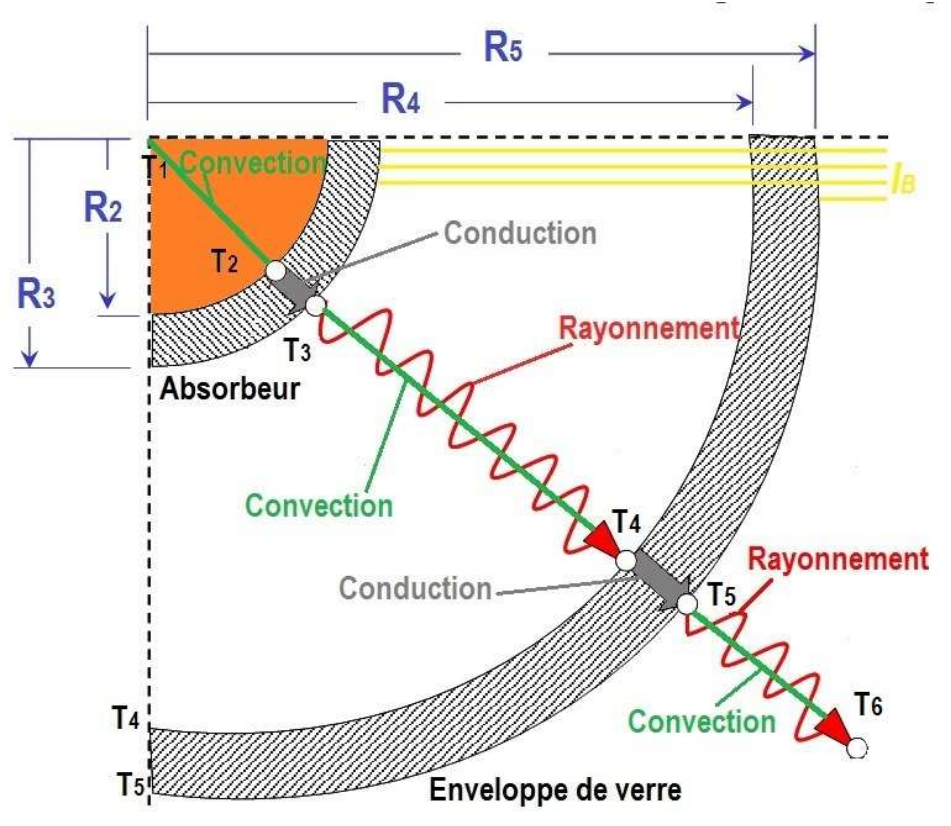

Fig.5. Phénomènes de transfert thermique dans le récepteur solaire 


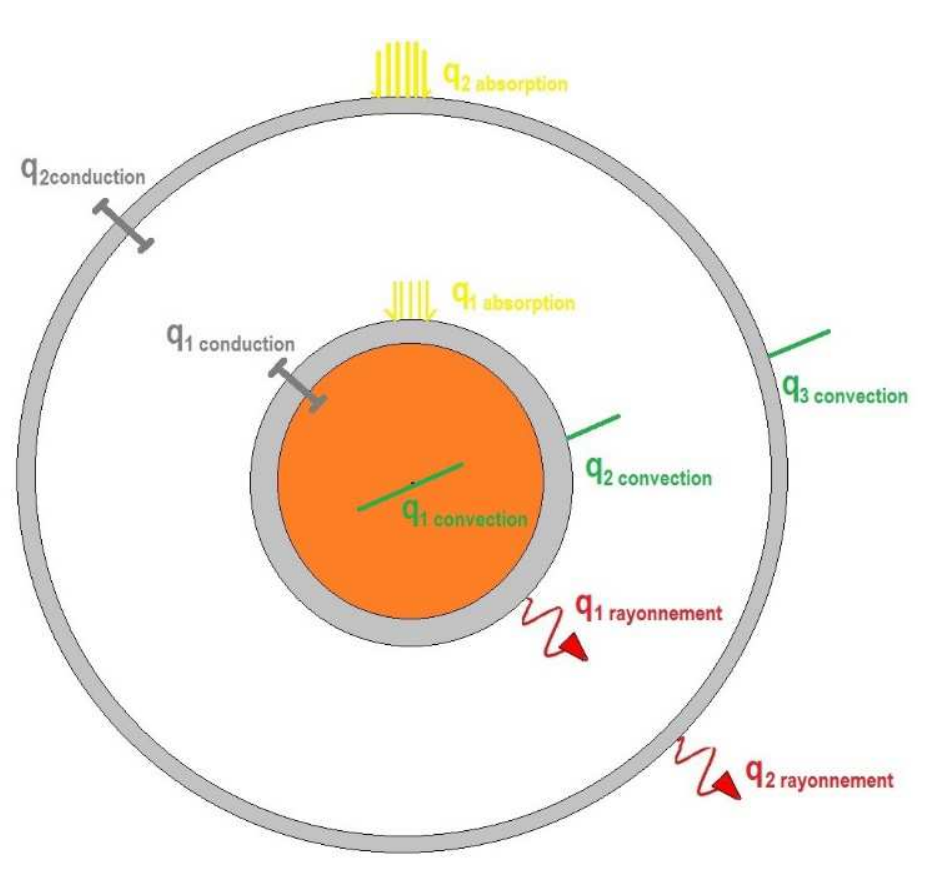

Fig.6. Bilan énergétique du modèle 1D du concentrateur étudié

Transfert de chaleur par convection à partir de l'enveloppe de verre à l'atmosphère

Le transfert de chaleur par convection à la surface externe de l'enveloppe de verre (couverture) à l'atmosphère peut être changé en fonction des conditions du vent à l'air ambiant. L'estimation du transfert de chaleur par convection nécessite le calcul du coefficient de transfert de chaleur moyen sur toute la surface, bien que la variation du coefficient de transfert de chaleur local tout le long de la périphérie de couverture n'est pas uniformément distribuée. Le nombre de Nusselt $\mathrm{Nu}$ peut être obtenu en utilisant l'équation suivante:

$$
h_{a i r}=N u_{a i r}: \frac{k_{\text {air }}}{D_{\mathrm{s}}}
$$

$h_{\text {air }}$ Coefficient de transfert thermique convectif de l'air ambient $\left(\mathrm{W} / \mathrm{m}^{2} . \mathrm{K}\right)$

$N u_{\text {air }}$ Nombre de Nusselt à la température de l'air.

$k_{\text {air }}$ Conductivité thermique de l'air (W/m.K).

$D_{5}$ Diamètre externe de l'enveloppe de verre (m).

Le nombre de Nusselt nécessite de savoir si le transfert de chaleur par convection est naturel ou forcé. S'il y a du vent, le transfert de chaleur sera par convection forcée. Donc la corrélation développée par Zhukauskas sera utilisée pour estimer le nombre de Nusselt [24]: 


$$
N u_{a i r^{\prime}}=C \cdot R e_{a i r^{*}}^{m} P r_{a i r^{*}}^{n}\left(\frac{p_{Y_{6}}^{n}}{\dot{P}_{Y_{5}}^{n}}\right)^{1 / 4}
$$

Les paramètres de corrélation sont donnés dans le tableau 2.

Le terme exponentiel est donnée par:

$\mathrm{n}=0.37$ pour $\operatorname{Pr} \leq 10$

$\mathrm{n}=0.36$ pour $\operatorname{Pr}>10$.

Avec: $P r={ }^{\mu \cdot C_{p}} / k$

$\vec{p} T_{T_{\text {air }}}$ Nombre de Prandtl à $T_{6}$.

$\dot{P}_{r_{\text {air }}}$ Nombre de Prandtl à $T_{\mathrm{5}}$.

Tableau 2. Paramètres de corrélation [24]

\begin{tabular}{|c|c|c|}
\hline $\operatorname{Re}$ & C & $m$ \\
\hline $1-40$ & 0,750 & 0,4 \\
\hline $40-1000$ & 0,510 & 0,5 \\
\hline $1000-200.000$ & 0,260 & 0,6 \\
\hline $200.000-1.000 .000$ & 0,076 & 0,7 \\
\hline
\end{tabular}

Donc, l'expression de la puissance de chaleur convective transférée dans l'atmosphère par unité de longueur de récepteur est:

$$
\dot{q}_{3 \text {-convection }}=h_{a i r} \pi D_{5}\left(T_{5}-T_{6}\right)
$$

Transfert de chaleur par rayonnement de l'enveloppe de verre à l'atmosphère

Pour le transfert de chaleur externe par rayonnement, l'enveloppe de verre est considérée comme un objet gris convexe dans une grande cavité de corps noir, de façon que l'échange par rayonnement soit :

$$
\dot{q}_{\text {2-rayonnement }}=\sigma \pi D_{\mathrm{5}} \mathrm{E}_{\text {werre }}\left(T_{\mathrm{E}}^{4}-T_{\text {oiel }}^{4}\right)
$$

Où $\sigma$ est la constante de Stefan-Boltzmann, et $\varepsilon_{\text {verre }}$ est l'émissivité de l'enveloppe de verre. La température du ciel est généralement établie à $8^{\circ} \mathrm{C}$ inférieure à la température ambiante.

Transfert de chaleur par convection entre l'absorbeur et l'enveloppe de verre

Le processus de transfert de chaleur entre ces deux éléments par convection dépend 
directement de la pression à l'intérieur de l'espace annulaire. Lorsque la pression dans l'espace annulaire est assez faible ( $<1$ torr ou $133 \mathrm{~Pa}$ ), le transfert de chaleur se produit en raison de la conduction moléculaire. Pour des pressions supérieures à la valeur précédente, la convection naturelle prend place [25].

En considérant qu'il y a une bonne qualité de vide dans l'espace annulaire du tube récepteur de notre système, le transfert de chaleur par convection survient donc par une conduction moléculaire libre:

$$
\dot{q}_{2 \text {-convection }}=\pi D_{3} h_{2}\left(T_{3}-T_{4}\right)
$$

Avec:

$$
\begin{aligned}
& h_{2}=\frac{k_{a s p}}{\left(D_{3} / 2 \ln \left(D_{4} / D_{3}\right)+b \lambda\left(D_{3 / D_{4}+1}\right)\right)} \\
& b=\frac{(2-a)(9 \gamma-5)}{2 a(\gamma+1)} \\
& \lambda=\frac{2.331 e(-20)\left(T_{34}+273.15\right)}{\left(P_{a} \delta^{2}\right)}
\end{aligned}
$$

Où $a$ représente le coefficient d'accommodation, $b$ est un coefficient d'interaction, $k_{e s p}$ est la conductivité thermique du gaz à l'intérieur de l'espace annulaire à une température et une pression standard ${ }_{F} \lambda$ est le moyen chemin libre entre les collisions d'une molécule $(\mathrm{cm}), \gamma$ est le rapport des chaleurs spécifiques pour l'espace annulaire de l'air, $\delta$ ' est le diamètre moléculaire de l'espace annulaire de l'air, et $\mathrm{T}_{34}$ est la température moyenne des surfaces 3 et 4. Selon [25] $h_{2}=0.0001115 \mathrm{~W} / \mathrm{m}^{2} \mathrm{~K}$.

\section{Transfert de chaleur par rayonnement entre l'absorbeur et l'enveloppe de verre}

La partie majeure du transfert de chaleur entre l'absorbeur et l'enveloppe de verre est due à l'échange thermique radiatif entre ces deux surfaces. Selon [24], le transfert thermique par rayonnement peut être exprimé comme suit: 


$$
\dot{q}_{1-\text { rayonnement }}=\frac{\sigma \pi D_{3}\left(T_{3}^{4}-T_{4}^{4}\right)}{\left(1 / s_{3}+\left(1-\varepsilon_{4}\right) D_{3} /\left(\varepsilon_{4} D_{4}\right)\right)}
$$

> Transfert de chaleur par conduction à travers l'enveloppe de verre

L'équation de transfert de chaleur par conduction à travers l'enveloppe de verre est l'équation commune de Fourier pour un cylindre avec une conductivité thermique $\mathrm{k}_{\mathrm{verre}}=1,04 \mathrm{~W} / \mathrm{m} \mathrm{K}$.

$$
\dot{q}_{2 \text {-conduction }}=2 \pi k_{\text {verre }}\left(T_{4}-T_{5}\right) / \ln \left(D_{5} / D_{4}\right)
$$

\section{Transfert de chaleur par conduction à travers le tube absorbeur}

L'expression est identique au cas de la conduction à travers l'enveloppe de verre, la conductivité thermique de l'acier est fixée à $\mathrm{k}_{\mathrm{acier}}=25 \mathrm{~W} / \mathrm{m} \mathrm{K}$.

$$
\dot{q}_{1 \text {-conduction }}=2 \pi k_{\text {acier }}\left(T_{2}-T_{3}\right) / \ln \left(D_{3} / D_{2}\right)
$$

\section{Transfert de chaleur par convection au fluide caloporteur}

Le transfert de chaleur par convection entre le tube absorbeur et le fluide caloporteur (soit l'huile thermique ou le sel fondu) qui circule à l'intérieur de tube a été calculé en mettant en œuvre la corrélation de Gnielinsky dans notre modèle [24]:

$$
q_{1-\text { convection }}=h_{F T C} D_{2} \pi\left(T_{2}-T_{F T C}\right)
$$

Où $h_{F T C}$ est estimé comme suit :

$$
\begin{aligned}
& h_{F T C}=N u_{D_{2}} \frac{k_{F T C}}{D_{2}} \\
& N u_{D_{2}}=\frac{f_{2} / 8\left(R e_{D_{2}}-1000\right) P r_{1}}{1+12.7 \sqrt{f_{2} / 8}\left(P r_{1}^{2 / 3}-1\right)}\left(\frac{P r_{1}}{P r_{2}}\right)^{0.11} \\
& f_{2}=\left(1.82 \log \left(R e_{D_{2}}\right)-1.64\right)^{-2}
\end{aligned}
$$

Le paramètre $k_{F T c}$ représente la conductivité thermique du FTC à la température $\mathrm{T}_{\mathrm{FTC}}, f_{2}$ est le facteur de friction. Les indices 1 et 2 pour les nombres de Prandtl sont déterminés à la température à laquelle ils sont évalués.

$>$ Absorption du rayonnement solaire par l'enveloppe de verre et l'absorbeur

L'énergie solaire absorbée par la surface de l'enveloppe de verre et l'absorbeur a été déjà calculée et sa distribution a été déjà simulée dans la section 2.2.4. 
La résolution du problème mathématique a été effectuée en utilisant la méthode de Newton avec une précision de $0.001^{\circ} \mathrm{C}$, depuis les équations formant un système non linéaire. Un système général d'équations de:

$$
F(X)=0\left\{\begin{array}{c}
F: \mathbb{R}^{n} \rightarrow \mathbb{R}^{n} \\
\text { et } \\
x=\left(x_{1}, \cdots, x_{n}\right) \rightarrow\left(f_{1}(x), \cdots, f_{n}(x)\right)
\end{array}\right.
$$

Peut être résolu en utilisant la méthode de Newton. Cela signifie que l'approximation de la valeur de la fonction soit donnée par l'expression suivante:

$$
F(x) \cong F\left(x^{(0)}\right)+D F\left(x^{(0)}\right) \cdot\left(x-x^{(0)}\right)
$$

A l'étape dite de Newton qui a l'aspect suivant:

$$
X^{(1)}=X^{(0)}-D F\left(X^{(0)}\right)^{-1} \cdot F\left(X^{(0)}\right)
$$

Si les équations (23), (24), (25) et (26) peuvent être représentées en substituant les termes du flux de chaleur par leurs expressions respectives, on obtient des équations exprimées en fonction de chaque température nodale de notre régime de résistance thermique.

$$
\begin{aligned}
& h_{F T C} D_{2} \pi\left(T_{2}-\frac{T_{\text {entré }}}{2}-\frac{T_{\text {sortie }}}{2}\right)-2 \pi k_{\text {aoter }}\left(T_{2}-T_{3}\right) / n\left(D_{3} / D_{2}\right)=0 \\
& \dot{q}_{1 \text {-absorption solaire }}-\pi D_{3} h_{2}\left(T_{3}-T_{4}\right)-\Psi\left(T_{3}^{4}-T_{4}^{4}\right)-2 \pi k_{\text {aoier }}\left(T_{2}-T_{3}\right) / \ln \left(D_{3} / D_{2}\right) \\
& =0 \\
& \pi D_{3} h_{2}\left(T_{3}-T_{4}\right)-\psi\left(T_{3}^{4}-T_{4}^{4}\right)-2 \pi k_{\text {verre }}\left(T_{4}-T_{5}\right) / \ln \left(D_{5} / D_{4}\right)=0
\end{aligned}
$$

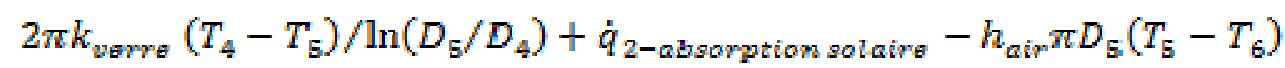

$$
\begin{aligned}
& -\sigma \pi D_{5} \varepsilon_{\text {werre }}\left(T_{5}^{4}-T_{\text {oitl }}{ }^{4}\right)=0
\end{aligned}
$$

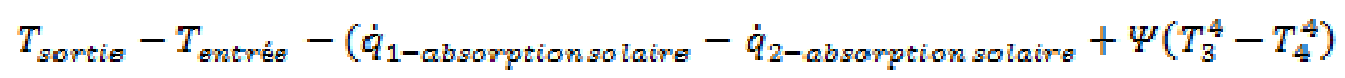

$$
\begin{aligned}
& \left.+\pi D_{3} h_{2}\left(T_{3}-T_{4}\right)\right)^{L} / m_{F T C} C_{p_{F T C}}=0
\end{aligned}
$$

Le Jacobien de cette série de cinq équations doit être calculé afin de mettre en œuvre la méthode de Newton: 
$J=\left(\begin{array}{ccc}\frac{\partial y_{1}}{\partial x_{1}} & \cdots & \frac{\partial y_{n}}{\partial x_{n}} \\ \vdots & \cdots & \vdots \\ \frac{\partial y_{m}}{\partial x_{1}} & \cdots & \frac{\partial y_{m}}{\partial x_{n}}\end{array}\right) \quad J=\left(\begin{array}{ccc}j_{11} & \cdots & j_{15} \\ \vdots & \cdots & \vdots \\ j_{51} & \cdots & j_{55}\end{array}\right)$

Le Jacobien de notre série d'équations est alors:

$j_{11}=-h_{F T C} D_{2} \pi / 2$

$j_{12}=h_{F T C} D_{2} \pi-2 \pi k_{\text {aciar }} / \ln \left(D_{3} / D_{2}\right)$

$j_{13}=2 \pi k_{\text {aeier }} / \ln \left(D_{3} / D_{2}\right)$

$j_{22}=-2 \pi k_{\text {aciar }} / \ln \left(D_{3} / D_{2}\right)$

$j_{2 g}=-2 \pi D_{3} h_{2}-4 \Psi T_{3}^{3}+2 \pi k_{\text {acier }} / \ln \left(D_{3} / D_{2}\right)$

$j_{24}=\pi D_{3} h_{2}-4 \Psi T_{4}^{3}$

$j_{31}=1$

$j_{3 a}=\left(4 \Psi T_{3}^{3}+\pi D_{3} h_{2}\right) L / m_{F T C} C_{p_{F T C}}$

$j_{34}=\left(-4 \Psi T_{4}^{3}-\pi D_{3} h_{2}\right) L / m_{F T C} C_{p_{F T C}}$

$j_{43}=4 \Psi T_{4}^{3}+\pi D_{3} h_{2}$

$j_{44}=-4 \Psi_{4}^{3}-\pi D_{3} h_{2}-2 \pi k_{\text {varra }} / \ln \left(D_{\mathrm{B}} / D_{4}\right)$

$j_{45}=2 \pi k_{\text {verre }} / \ln \left(D_{5} / D_{4}\right)$

$j_{54}=2 \pi k_{\text {varre }} / \ln \left(D_{5} / D_{4}\right)$

$j_{\mathrm{ES}}=-2 \pi k_{\text {verre }} / \ln \left(D_{\mathrm{g}} / D_{4}\right)-h_{3} \pi D_{\mathrm{s}}-4 \sigma D_{\mathrm{E}} \pi \varepsilon_{\mathrm{g}} T_{\mathrm{E}}^{3}$

$j_{14}=j_{15}=j_{12}=j_{25}=j_{32}=j_{35}=j_{41}=j_{42}=j_{51}=j_{52}=j_{53}=0$

L'étape comme décrite dans l'équation 44 aura l'expression suivante qui a besoin des valeurs initiales pour les inconnues afin de démarrer la procédure de résolution itérative. 


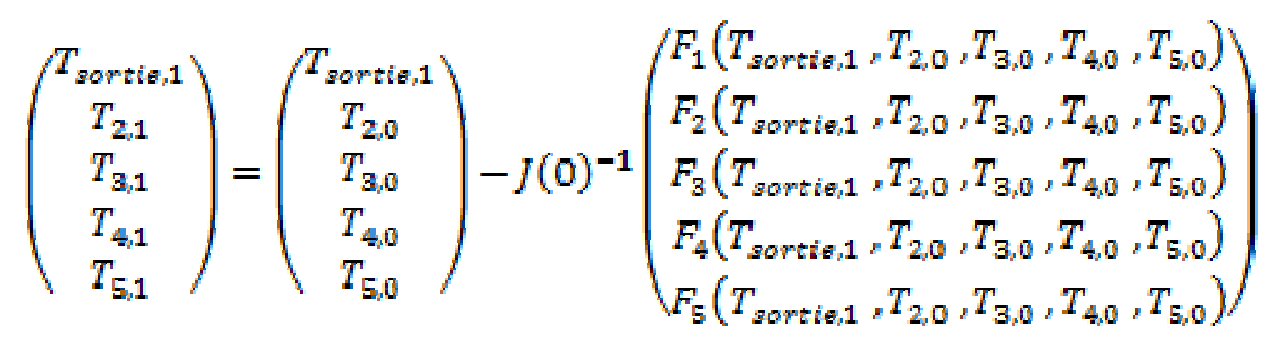

\subsection{Validation}

Le rendement optique de 75,2\% avec une densité de flux de chaleur moyenne calculée du capteur de $15977,3 \mathrm{~W} / \mathrm{m}^{2}$ peut être obtenu par l'équation classique:

$$
\eta_{\text {opt }}=\frac{q_{\mathrm{u}} \pi D_{3} L}{I_{B} A_{m}}
$$

La référence [10] montre que les résultats expérimentaux de l'efficacité optique sont d'environ $73,7 \%$. Par conséquent, il y a un bon accord de 2,03\% entre la simulation et les résultats expérimentaux.

Pour la performance thermique de tube récepteur, les équations élaborées du modèle ont été programmés à l'aide de Matlab. Les résultats du modèle de transfert de chaleur développé ont été comparés avec les résultats des essais expérimentaux du récepteur solaire de même type [11] à l'aide de sept paramètres statistiques (Annexe I). Dans cette configuration où les tests ont été effectués, Il n'y a pas de rayonnement solaire incident sur le recepteur, et aucun FTC ne circule à l'intérieur du tube. Au lieu de cela, la surface intérieure de l'absorbeur est chauffée électriquement pour maintenir une température constante. Les données disponibles incluent la perte de chaleur mesurée pour les différentes températures de la surface intérieure du tube absorbeur et de la température de la surface extérieure de l'enveloppe de verre. Comme le montre la figure 8 , et le tableau 3, le modèle présente une bonne corrélation avec les résultats expérimentaux. Le petit écart dans le modèle actuel par rapport au travail expérimental est présenté dans la référence [11], principalement due à l'approximation utilisée pour calculer les coefficients de perte de chaleur.

Tableau 3. Validation des résultats de simulation avec le modèle et les essais expérimentaux

\begin{tabular}{cccccccc}
\hline & $\boldsymbol{M P E}$ & $\boldsymbol{M A P E}$ & $\boldsymbol{M B E}$ & $\boldsymbol{M A B E}$ & $\boldsymbol{R M S E}$ & $\boldsymbol{R}^{2}$ & $\boldsymbol{t}_{\text {sta }}$ \\
\hline $\begin{array}{c}\text { Modèle avec } \\
\text { l'expérimental }\end{array}$ & $-6,8843$ & 6,8843 & $-13,5833$ & 13,5833 & 18,5775 & 0,9960 & 3,5547 \\
\hline
\end{tabular}




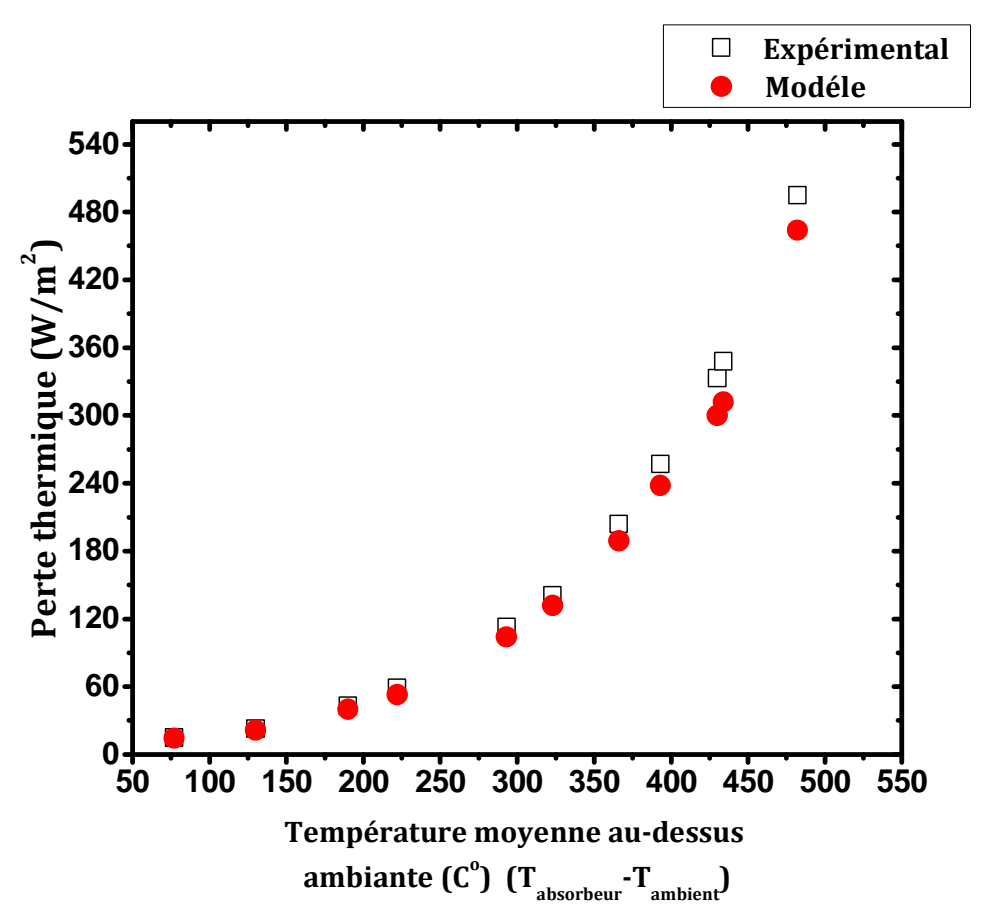

Fig.8. Validation du modèle du récepteur solaire

\section{RÉSULTATS ET DISCUSSION}

Les données horaires du rayonnement solaire, ainsi que la température ambiante et la vitesse du vent sont des paramètres clés pour simuler les performances du concentrateur solaire. À cet effet, les données de Béchar (Algérie) avec une série de valeurs d'intervalle d'une heure ont été choisies. Les variations horaires moyennes du modificateur de l'angle d'incidence et de la perte d'extrémité du concentrateur solaire avec un réflecteur de type Solargenix LS-2 et un tube récepteur de type Schott PTR 2008, sont présentées dans les figures 9.a (Juin) et 9.b (Décembre). Par ailleurs, la variation des performances optiques du concentrateur solaire de même type est présentée dans la figure 10. Comme nous utilisons le même type de réflecteur

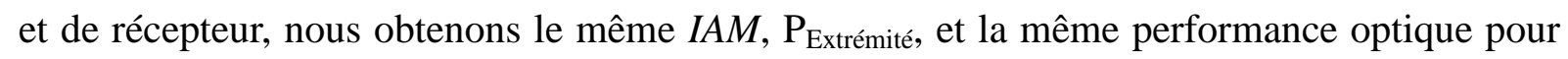
les deux concentrateurs avec les deux fluides (huile et sel). Un autre point très important est la différence dans les efficacités optiques $\left(I A M, P_{\text {Extrémité }}, \eta_{\text {opt }}\right)$ entre Juin et Décembre, il est principalement dû à la différence dans la hauteur angulaire du soleil (altitude solaire) et l'angle de zénith qui prend des valeurs élevées en été, et faibles en hiver, ainsi l'augmentation dans ces deux angles entraîne une augmentation des efficacités optiques. 
(a)

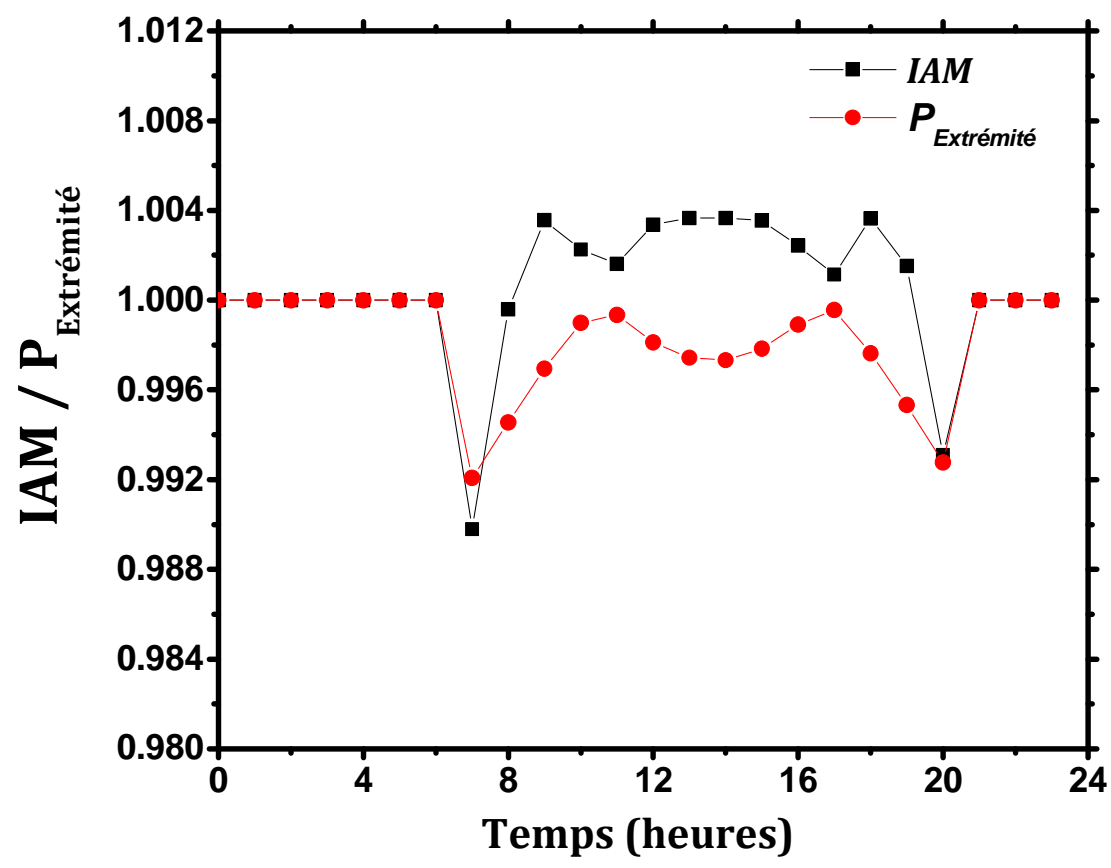

(b)

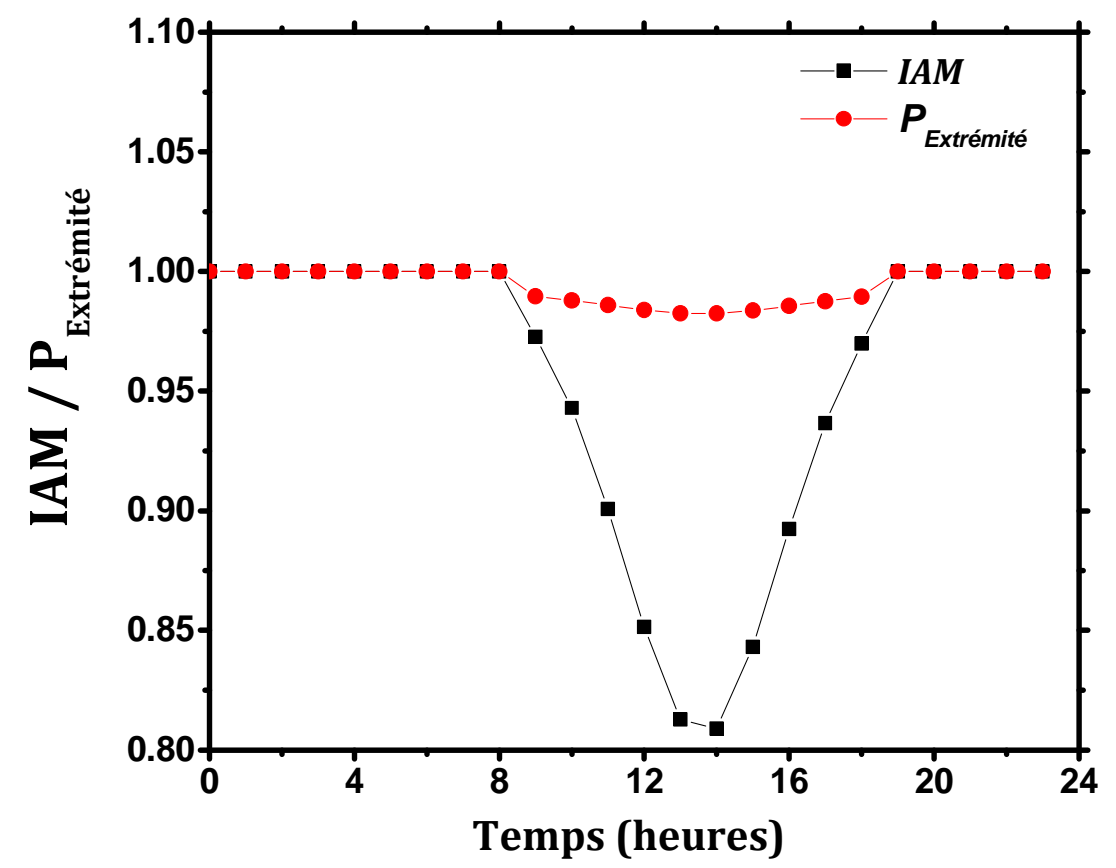

Fig.9. Variations horaires moyennes de IAM et de $\mathrm{P}_{\text {Extrémité }}$ en (a) Juin et (b) Décembre du concentrateur solaire 


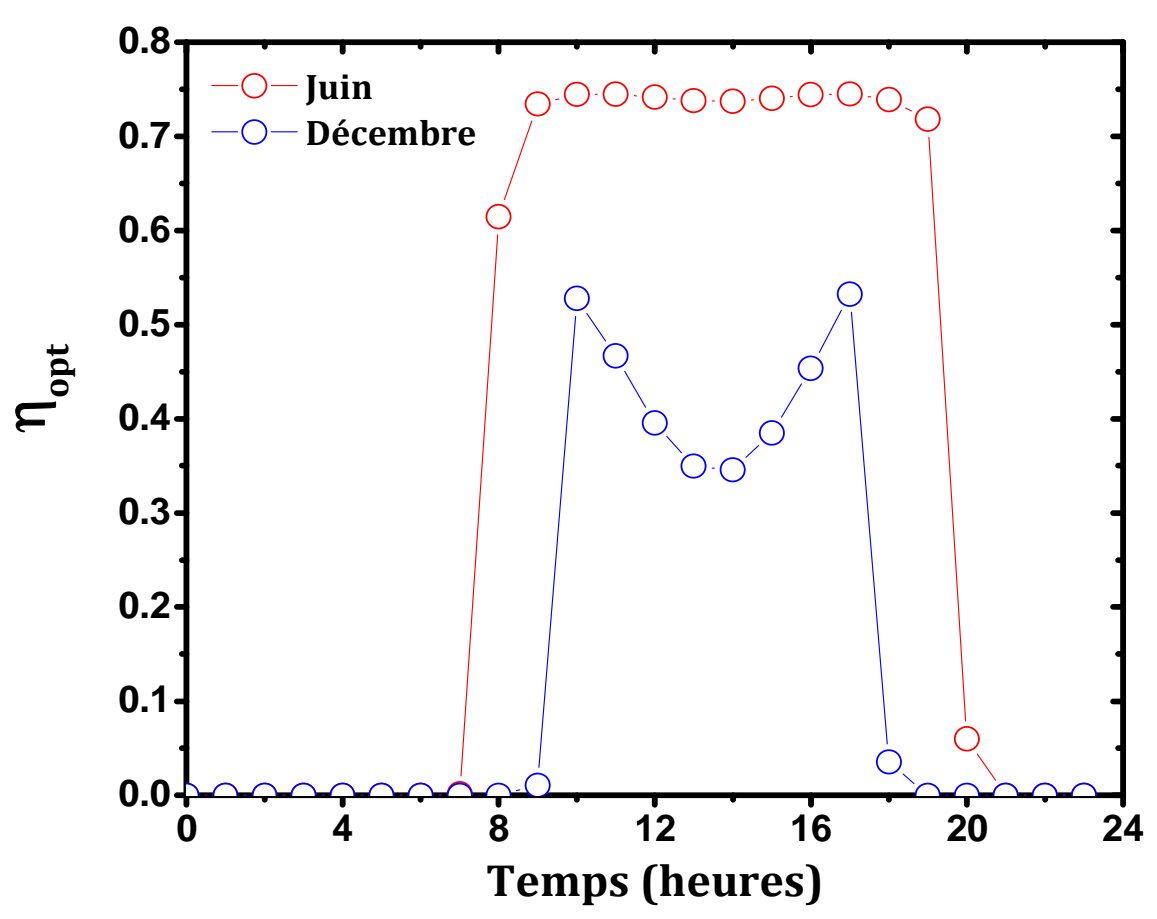

Fig.10. Variation des performances optiques horaires moyennes en Juin et Décembre du concentrateur solaire

La figue 11 montre la comparaison des performances totales horaires moyennes durant les deux mois de l'année (Juin et Décembre) du concentrateur solaire, avec les deux fluides caloporteurs. Il en résulte que le choix de l'huile comme fluide de travail dans le tube récepteur est le plus recommandé par rapport au sel. Le premier fluide donne des performances totales maximales jusqu'à $67 \%$ en Juin et $48 \%$ en Décembre, tandis que le deuxième fluide montre des performances baisses par rapport au premier avec des performances totales maximales de $63 \%$ en Juin et $44 \%$ en Décembre.

Les variations des performances totales du concentrateur avec les deux fluides, même si celles optiques sont identiques (Fig 10), sont dues à la différence dans les gammes des températures de travail. En conséquence, la perte thermique du sel est supérieure à celle de l'huile. Mais d'autre part, cette élévation de la température du sel provoque une augmentation de la performance du cycle de production d'énergie. 

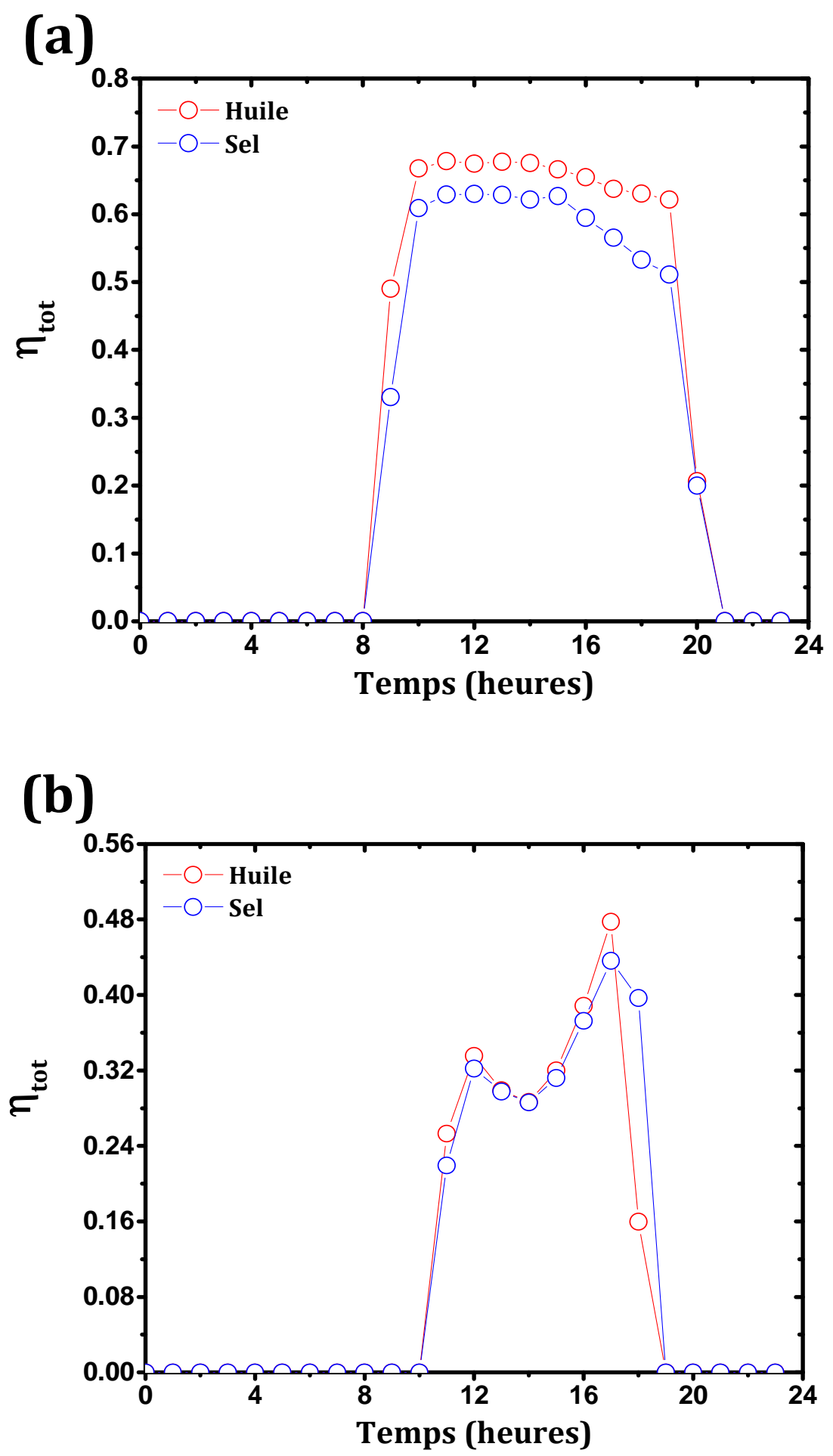

Fig.11. Comparaison des performances totales horaires moyennes en (a) Juin et (b) Décembre du concentrateur en utilisant deux fluides (huile et sel)

\section{CONCLUSION}

Dans cette étude, nous avons proposé une simulation des performances optique et thermique 
d'un concentrateur solaire cylindro-parabolique avec un réflecteur de type Solargenix LS-2 et un tube récepteur de type Schott PTR 2008, et qui utilise deux fluides caloporteurs différents: l'huile thermique de type Therminol VP1 et le sel fondu.

Cette étude favorise le choix de l'huile comme fluide caloporteur dans le tube récepteur plus que l'utilisation du sel fondu. En outre, le concentrateur solaire avec l'huile thermique montre une meilleure performance totale que celle utilisant le sel, même s'ils ont la même performance optique. Le premier fluide donne des performances totales maximales jusqu'à $67 \%$ et $48 \%$ en Juin et Décembre respectivement, tandis que le deuxième fluide montre des performances baisses par rapport au premier avec des performances totales maximales de $63 \%$ (Juin) et $44 \%$ (Décembre).

\section{ANNEXES}

\section{Annexe I. Paramètres d'évaluation statistique des erreurs}

Dans ce travail, sept indicateurs ou paramètres d'évaluation statistique ont été utilisés pour les comparaisons et validations, à savoir: le coefficient de détermination $\left(R^{2}\right)$, l'erreur moyenne en pourcentage (MPE), l'erreur absolue moyenne en pourcentage (MAPE), le biais d'erreur moyenne $(M B E)$, le biais absolu d'erreur moyenne $(M A B E)$, la racine carrée de l'erreur quadratique moyenne (RMSE), et le test statistique $t_{\text {sta }}$.

$$
\begin{aligned}
& R^{2}=\frac{\sum_{i=1}^{n}\left(Y_{i, o}-Y_{i, a}\right)^{2}}{\sum_{i=1}^{n}\left(Y_{i, o}-\bar{Y}_{i, o}\right)^{2}} \\
& M P E=\frac{1}{n} \sum_{i=1}^{n}\left(\frac{Y_{i, o}-Y_{i, e}}{Y_{i, \sigma}}\right) \times 100 \\
& M A P E=\frac{1}{n} \sum_{i=1}^{n}\left(\left|\frac{Y_{i, o}-Y_{i, \sigma}}{Y_{i, o}}\right|\right) \times 100 \\
& M B E=\frac{1}{n} \sum_{i=1}^{n}\left(Y_{i, \sigma}-Y_{i, a}\right) \\
& M A B E=\frac{1}{n} \sum_{i=1}^{n}\left(\left|Y_{i o}-Y_{i, e}\right|\right)
\end{aligned}
$$




$$
\begin{aligned}
& R M S E=\frac{1}{n} \sqrt{\sum_{i=1}^{n}\left(Y_{i, o}-Y_{i, d}\right)^{2}} \\
& t_{s t a}=\sqrt{\left[\frac{(n-1) M B E^{2}}{R M S E^{2}-M B E^{2}}\right]}
\end{aligned}
$$

Avec $Y_{i, m}, Y_{i, q}, \bar{Y}_{i, m}$ et $\mathrm{n}$ les valeurs observées, calculées, la moyenne des valeurs observées et le nombre d'observations, respectivement.

\section{Annexe II. Propriétés thermo-physiques des fluides de transfert de chaleur}

La masse volumique $\rho\left(\mathrm{kg} / \mathrm{m}^{3}\right)$ est définie par:

$$
p=\sum_{i=0}^{N} a_{i} T^{i}
$$

Avec T en $\left({ }^{\circ} \mathrm{C}\right)$, et $a_{i}$ les coefficients des corrélations (tableau A2.1)

Tableau A2.1. Coefficients des corrélations de masse volumique

\begin{tabular}{c|c|c|c|c}
\hline \multicolumn{1}{c|}{$\boldsymbol{F T C}$} & $\boldsymbol{a}_{\boldsymbol{0}}$ & $\boldsymbol{a}_{\boldsymbol{1}}$ & $\boldsymbol{a}_{\mathbf{2}}$ & $\boldsymbol{a}_{\mathbf{3}}$ \\
\hline Therminol VP-1 [1] & 1083,22 & $-0,902$ & $7,369.10^{-4}$ & $-2,287.10^{-6}$ \\
\hline Sel Fondu [2] & 2090,18 & $-0,640$ & 0 & 0 \\
\hline
\end{tabular}

La capacité thermique à pression constante $(\mathrm{kJ} / \mathrm{kg} . \mathrm{K})$ est donnée par:

$$
c_{p}=\sum_{i=0}^{N} b_{i} T^{i}
$$

Avec T en $\left({ }^{\circ} \mathrm{C}\right)$, et $b_{i}$ les coefficients des corrélations (tableau A2.2)

Tableau A2.2. Coefficients des corrélations de capacité thermique

\begin{tabular}{c|c|c|c|c|c}
\hline \multicolumn{1}{c|}{$\boldsymbol{F T C}$} & $\boldsymbol{b}_{\boldsymbol{0}}$ & $\boldsymbol{b}_{\boldsymbol{1}}$ & $\boldsymbol{b}_{\boldsymbol{2}}$ & $\boldsymbol{b}_{\boldsymbol{3}}$ & $\boldsymbol{b}_{\boldsymbol{4}}$ \\
\hline Therminol VP-1 [1] & 1,471 & $3,497.10^{-3}$ & $-4,817.10^{-6}$ & $8,400.10^{-9}$ & 0 \\
\hline Sel Fondu [2] & 1,093 & $3,755.10^{-3}$ & $-1,322.10^{-5}$ & $2,112.10^{-8}$ & $-1,2.10^{-11}$ \\
\hline
\end{tabular}

L'enthalpie spécifique $(\mathrm{kJ} / \mathrm{kg})$ est donnée par:

$$
h=\sum_{i=0}^{N} c_{i} T^{i}
$$


Avec T en $\left({ }^{\circ} \mathrm{C}\right)$, et $c_{i}$ les coefficients des corrélations (tableau A2.3).

Tableau A2.3. Coefficients des corrélations d'enthalpie spécifique

\begin{tabular}{l|c|c|c|c|c|c}
\hline \multicolumn{1}{c|}{$\boldsymbol{F T C}$} & $\boldsymbol{c}_{\boldsymbol{0}}$ & $\boldsymbol{c}_{\boldsymbol{1}}$ & $\boldsymbol{c}_{\boldsymbol{2}}$ & $\boldsymbol{c}_{\boldsymbol{3}}$ & $\boldsymbol{c}_{\boldsymbol{4}}$ & $\boldsymbol{c}_{\boldsymbol{5}}$ \\
\hline Therminol VP-1 $[1]$ & $-18,977$ & 1,513 & $1,2908.10^{-3}$ & $1,201.10^{-7}$ & 0 & 0 \\
\hline Sel Fondu $[2]$ & $-354,845$ & 1,092 & $1,877.10^{-3}$ & $-4,409.10^{-6}$ & $5,282.10^{-9}$ & $-2,4.10^{-12}$ \\
\hline
\end{tabular}

La conductivité thermique (W/m.K) est présentée par:

$$
k=\sum_{i=0}^{N} d_{i} T^{i}
$$

Avec T en ( $\left.{ }^{\circ} \mathrm{C}\right)$, et $d_{i}$ les coefficients des corrélations (tableau A2.4).

Tableau A2.4. Coefficients des corrélations de conductivité thermique

\begin{tabular}{l|c|c|c}
\hline \multicolumn{1}{c|}{$\boldsymbol{F T C}$} & $\boldsymbol{d}_{\boldsymbol{0}}$ & $\boldsymbol{d}_{\boldsymbol{1}}$ & $\boldsymbol{d}_{\boldsymbol{2}}$ \\
\hline Therminol VP-1 [1] & 0,138 & $-8,738.10^{-5}$ & $-1,720.10^{-7}$ \\
\hline Sel Fondu [2] & 0,441 & $1,953.10^{-4}$ & 0 \\
\hline
\end{tabular}

La viscosité dynamique (mPa.s) est présentée pour l'huile Therminol VP-1 comme suit:

$$
\ln \mu=\sum_{i=0}^{N} e_{i} T^{i}
$$

Et pour le sel fondu comme suit:

$$
\mu=\sum_{i=0}^{N} e_{i} T^{i}
$$

Avec T en $\left({ }^{\circ} \mathrm{C}\right)$, et $e_{i}$ les coefficients des corrélations (tableau A2.5)

Tableau A2.5. Coefficients des corrélations de viscosité dynamique

\begin{tabular}{l|c|c|c|c|c}
\hline \multicolumn{1}{c|}{$\boldsymbol{F T C}$} & $\boldsymbol{e}_{\boldsymbol{0}}$ & $\boldsymbol{e}_{\boldsymbol{1}}$ & $\boldsymbol{e}_{2}$ & $\boldsymbol{e}_{3}$ & $\boldsymbol{e}_{\boldsymbol{4}}$ \\
\hline Therminol VP-1 $[1]$ & 2,008 & $-2,989.10^{-2}$ & $1,207.10^{-4}$ & $-2,714.10^{-7}$ & $2,370.10^{-10}$ \\
\hline Sel Fondu $[2]$ & 22,713 & $-1,200.10^{-1}$ & $2,281.10^{-4}$ & $-1,474.10^{-7}$ & 0 \\
\hline
\end{tabular}

\section{RÉFÉRENCES}

[1] Patnode A M. Simulation and performance evaluation of parabolic trough solar power plants. Université de Wisconsin-Madison, États Unis; 2006. Thèse de Master. 
[2] Li L, Wang Z F, Li J B, Lei D Q. A vacuum reliability analysis of parabolic trough receiver. Sol. Energy. Mater. Sol. Cells. 105, 2012, 302-308, doi:10.1016/j.solmat.2012.06.034

[3] Men W, Matthew R, Aldo S. Three-dimensional optical and thermal numerical model of solar tubular receivers in parabolic trough concentrators. J. Sol. Energy. Eng. Trans. 134(4), 041012-1, doi:10.1115/1.4007494

[4] Liu Q, Wang Y L, Lei J, Jin H G. Modeling and optimizing parabolic trough solar collector systems using the least squares support vector machine method. Sol. Energy. 87(7), 2012, 1973-1980, doi:10.1016/j.solener.2012.01.026

[5] Marif Y, et al. Numerical simulation of solar parabolic trough collector performance in the Algeria Saharan region. . Energy. Convers. Manage. 85, 2014, 521-529, doi:10.1016/j.enconman.2014.06.002

[6] Eck M, Feldhoff J F, Uhlig R. Thermal modelling and simulation of parabolic trough receiver tubes. Proceedings of the ASME $4^{\text {th }}$ International Conference on Energy Sustainability, 2010. Phoenix.

[7] Cheng Z D, et al. Numerical simulation of a parabolic trough solar collector with non-uniform solar flux conditions by coupling FVM and MCRT method. Sol. Energy. 86, 2012, 1770-1784. doi:10.1016/j.solener.2012.02.039

[8] He Y, Xiao J, Cheng Z, Tao Y. A MCRT and FVM coupled simulation method for energy conversion process in parabolic trough solar collector. Renew. Energy. 36, 2011, 976-985, doi:10.1016/j.renene.2010.07.017

[9] Xiao J, et al. Performance analysis of parabolic trough solar collector. J. Eng. Thermophys., 2009, 30(5), 729-733.

[10] Dudley V E, et al. Test Results: SEGS LS-2 Solar Collector. SAND94-1884. Sandia National Laboratories 1994 http://www.nrel.gov/csp/troughnet/pdfs/segs_ls2_solar_collector.pdf

[11] Burkholder F, Kutscher C. Heat Loss Testing of Schott's 2008 PTR70 Parabolic Trough Receiver, Technical Report, NREL/TP-550-45633, National Renewable Energy Laboratory, Golden, 2009. www.nrel.gov/docs/fy09osti/45633.pdf

[12] Besarati S M, Padilla R V, Goswami D Y, Stefanakos E. The Potential of Harnessing 
Solar Radiation in Iran: Generating Solar Maps and Viability Study of PV Power Plants. Renew. Energy. 53, 2013, 193-199, doi:10.1016/j.renene.2012.11.012

[13] Duffie J. A, Beckman W. A. Solar engineering of thermal process. $2^{\text {ème }}$ Édition. New York: John Wiley \& Sons, 1991.

[14] Mecibah M S, Boukelia T E, Tahtah R, Gairaa K. Introducing the best model for estimation the monthly mean daily global solar radiation on a horizontal surface (Case study: Algeria). Renew. Sust. Energy. Rev. 36, 2014, 194-202, doi:10.1016/j.rser.2014.04.054

[15] Boukelia T E, Mecibah M S, Meriche I E. General models for estimation of the monthly mean daily diffuse solar radiation (Case study: Algeria). Energy. Convers. Manage. 81, 2014, 211-219, doi:10.1016/j.enconman.2014.02.035

[16] Collares-Pereira M, Rabl A. The average distribution of solar radiation correlations between diffuse and hemispherical and between daily and hourly insolation values. Sol. Energy. 22(2), 1979, 155-164, doi:10.1016/0038-092X(79)90100-2

[17] Liu B, Jordan R. The interrelationship and characteristic distribution of direct, diffuse and total solar radiation. Sol. Energy. 4(3), 1960, 1-19, doi:10.1016/0038-092X(60)90062-1

[18] Kalogirou S A. Solar Thermal Collectors and Applications. Prog. Energy. Combust. Sci. 30(3), 2004, 231-295, doi:10.1016/j.pecs.2004.02.001

[19] Goswami D. Y, Kreith F, Kreider J. F. Principles of solar engineering. $2^{\text {ème }}$ Édition. Philadelphia: Taylor \& Francis, 2000.

[20] Lippke F. Simulation of the Part Load Behavior of a 30MWe SEGS Plant. Sandia National Laboratories, Albuquerque, SAND95-1293, 1995. DOI: 10.2172/95571

[21] Stuetzle T A. Automatic Control of the 30MWe SEGS VI Parabolic Trough Plant. Université de Wisconsin-Madison, États Unis; 2002. Thése de Master

[22] Wendelin T. A new optical modeling tool for concentrating solar optics. Proceedings of ISEC 2003: International Solar Energy Conference, Hawaii, USA, 2003.

[23] Moreno J L. Thermo-economic optimisation of a trough-tower combination thermal solar power plant. Universidad Pontificia de Comillas. Thése de Master 2011.

[24] Incropera F. P, DeWitt D. P. Fundamentals of Heat and Mass Transfer. $5^{\text {éme }}$ Edition. New York: John Wiley \& Sons, 2002. 
[25] Wagner M J, Blair N, Dobos A. A Detailed Physical Trough Model for NREL's Solar Advisor Model. SolarPACES, 2001. www.nrel.gov/docs/fy11osti/49368.pdf

[26] Solutia Inc. Therminol vp-1, vapor phase/liquid phase heat transfer fluid $54^{\circ} \mathrm{f}$ to $750^{\circ} \mathrm{f}$. Technical bulletin 7239115 c, 2008.

[27] Zavoico, AB. Solar Power Tower Design Basis Document. Tech. rep, Sandia National Laboratories, SAND2001-2100, 2001.

How to cite this article:

Boukelia T.E, Mecibah M.S and Laouafi A. Performance simulation of parabolic trough solar collector using two fluids (thermic oil and molten salt). J. Fundam. Appl. Sci., 2016, 8(2), 600-626. 